\title{
Sir Richard Doll
}

\author{
Silvia Franceschi ${ }^{(1)}$
}

$S^{\text {in }}$ ir Richard Doll falleció en Oxford a la edad de 92 años el 24 de Julio de 2005 a causa de un fallo cardiaco agudo. Había pasado por varias jubilaciones: de la presidencia de Regius Professor of Medicine en 1979; de Green College, el cual fundó y donde cinco años fue director; y de la Universidad de Oxford en 1983. Tras 22 años de su última jubilación "oficial", aún se complacía en escribir artículos científicos, dar conferencias y viajar largas distancias. Tres semanas después de su muerte hubiera cumplido 93 años trabajando día tras día.

Se le conoce sobre todo por su trabajo relacionado con el tabaco. Durante los años cuarenta y cincuenta varios investigadores examinaron la asociación entre el tabaquismo y el cáncer pulmonar. Mas Richard Doll siempre será vinculado con la demostración de que el fumar causa cáncer, al utilizar y redefinir las más importantes herramientas de la epidemiología moderna: los estudios de series de casos, de casos y controles y de cohorte. Richard Doll comenzó con el profesor Austin Bradford Hill su destacado estudio de cohorte sobre los doctores ingleses y el tabaquismo, y lo concluyó con Sir Richard Peto y la Dra. Sarah Darby. La colaboración entre todos ellos duró 50 años y en ese tiempo el mismo Doll dejó de fumar.

Gracias a este abordaje "de cohorte" también le fue posible revelar, además del cáncer, una serie de enfermedades relacionadas con el tabaquismo. En el reciente y último artículo de seguimiento de 50 años de los doctores ingleses se observó claramente que la mitad de los fumadores persistentes muere como consecuencia de su adicción. ${ }^{1}$ Sin embargo, los logros científicos de Doll fueron mucho más allá del taba- quismo e incluyeron el esclarecimiento de los efectos cancerígenos de la radiación, tanto ionizante (como cáncer pulmonar y por radares) como no ionizante (por ejemplo los campos electromagnéticos y la leucemia infantil), los coefectos de los anticonceptivos orales, y la eficacia de la suplementación con la vitamina $\mathrm{D}$ en la prevención de las fracturas de hueso en los ancianos. Alentó a la Agencia Internacional para la Investigación sobre Cáncer a apoyar y recopilar datos sobre los registros de cáncer en cinco continentes.

Demostró de la manera más clara que el cáncer no es sólo una consecuencia natural del envejecimiento, sino, en gran medida, un padecimiento evitable. El trabajo de Doll constató que el cáncer es una combinación de la naturaleza, el estilo de vida y el azar, y que los contaminantes ambientales producidos por el hombre no necesariamente son el principal culpable. Afirmó la importancia de la cuantificación de las causas de cáncer y en el libro que lleva el mismo nombre, que publicó junto con Richard Peto en $1981,{ }^{2}$ mencionó el hallazgo de que el tabaquismo es el doble de peligroso que la suma de todas las demás amenazas de cáncer más comunes.

Personalmente diré que durante el tiempo que permanecí en Oxford en los años ochenta lo observé trabajar incansablemente sin aparente esfuerzo, rodeado por tres generaciones de alumnos increíblemente brillantes y por su igualmente destacada esposa Joan, que murió en 2001. Richard y Joan Doll vivieron para ver el comienzo del siglo XXI, pasaron por dos guerras mundiales, y experimentaron los inmensos cambios sociales y médicos que siguieron a una pasión política y

(I) Agencia Internacional para la Investigación sobre Cáncer, Lyon, Francia. 
compasión humana incesantes. Siempre acudí a Richard Doll en busca de inspiración y consejo a lo largo de los últimos 20 años, y él nunca me defraudó. Frecuentemente no tenía yo que preguntar siquiera, sino tan sólo recordar sus palabras: "Cada problema tiene que superarse alcanzando primero un entendimiento integral de su naturaleza para entonces ponderar cuál es la forma más directa y simple de llegar a una respuesta". ${ }^{3}$

\section{Referencias}

I. Doll R, Peto R, Boreham J, Sutherland I. Mortality in relation to smoking: 50 years' observations on male British doctors. BMJ 2004;328:1519-1528.

2. Doll R, Peto R. The causes of cancer. Oxford: Oxford University Press, I98I.

3. Darby S.A conversation with Sir Richard Doll. Epidemiology 2003; 14:375-379. 